\title{
PROTOTIPO RÁPIDO PARA DOCENCIA Y ESTRATEGIAS QUIRÚRGICAS EN PEDIATRÍA
}

RAPID PROTOTYPE FOR TEACHING AND SURGICAL STRATEGIES IN PEDIATRICS

\author{
Víctor H Defagó ${ }^{1}$, Santiago Fernandez ${ }^{2}$, Silvio Chaile ${ }^{2}$, Hugo Robledo ${ }^{1}$, \\ Gabriel Massano ${ }^{2}$, Oscar Roldan ${ }^{1}$, Adriana Alday ${ }^{2}$
}

\section{Resumen:}

Introducción: La incorporación de la Clínica y la Imagenología permiten una mejor comprensión de la Anatomía. El objetivo de este trabajo es desarrollar un prototipo rápido en material sintético que replique detalles anatómicos para ser utilizado en la docencia y el entrenamiento quirúrgico en Pediatría.

Material y Método: Presentación de caso: Paciente de un año de edad con síndrome de dificultad respiratoria. En el examen endoscópico se halló una compresión traqueal distal. La angiotomografía confirmó la presencia de una malformación vascular. Con la finalidad de analizar una conducta adecuada, se solicitó la confección de un prototipo rápido a escala 1:1 que simulara una condición idéntica a la topografía torácica del paciente, utilizando imágenes virtuales 3D almacenadas en formato DICOM.

Técnica de generación de prototipo rápido: Se obtuvo una malla digital tridimensional y se generó el código "g" que se utilizó para controlar el hardware de producción. Se efectuó simulación digital y producción en material plástico (ABS) con técnica de deposición y fusión (MDF). Se validó el prototipo comparándolo con las mediciones testigos del modelo virtual en $3 \mathrm{D}$.

Resultados y Discusión: El modelo replicó exactamente los defectos hallados en la tomografía y endoscopía, confirmando la presencia de la malformación vascular y su repercusión sobre el aparato respiratorio. El prototipo rápido muestra las estructuras internas y externas del cuerpo humano con máxima precisión permitiendo una visión topográfica de situaciones "normales o patológicas" que facilitaría la docencia y el entrenamiento del equipo quirúrgico para proponer un plan de tratamiento adecuado. Hay numerosas áreas de la medicina que se beneficiarían con este modelo que podría ser construído con diversos tipos de materiales de diferente flexibilidad y consistencia.

Conclusiones: El prototipo rápido le da estado físico a las imágenes virtuales 3D, permitiendo la docencia y entrenamiento del equipo quirúrgico.

\section{Palabras clave: prototipo rápido, enseñanza de pediatría y anatomía}

\begin{abstract}
:
Introduction: The incorporation of the clinic and the imaging allow a better understanding of anatomy. The aim of this work is to develop a rapid prototype in synthetic material that replicates anatomical details to be used in teaching and surgical training in Pediatrics. Material and method: presentation of case: one year old female with respiratory distress syndrome. In the endoscopic examination was found a distal tracheal compression. The angiotomography confirmed the presence of a vascular malformation. In order to discuss appropriate conduct, the making of rapid prototyping in scale 1:1 was requested to simulate an identical condition of the thoracic topography of the patient, using virtual 3D images stored in the DICOM format. Rapid prototype technique: code "g" was generated, which was used to control the hardware of production and a three-dimensional digital grid was obtained. Digital simulation and production in plastic (ABS) with deposition and fusion technique (MDF) was performed. The prototype was validated by comparing measurements with witnesses of the virtual model in 3 D. Results and discussion: the model replied exactly the defects found in the scan and endoscopy, confirming the presence of vascular malformation and its impact on the respiratory system. Rapid prototype shows the internal and external structures of the human body with maximum precision allowing a topographic view of "normal or pathological" situations that could facilitate the teaching and training of the surgical team to propose an appropriate treatment plan. There are many areas of medicine that could benefit from this model that could be built with different types of materials with different flexibility and consistency.
\end{abstract}

Conclusions: Rapid prototype gives form to virtual 3D images, allowing teaching and training of the surgical team.

Key words: rapid prototype, teaching pediatric and anatomy

1. Cátedra de Clínica Pediátrica. Facultad de Ciencias Médicas.

2. Cátedra de Informática aplicada al diseño industrial. FAUD.UNC 


\section{Introducción}

En los últimos años, la enseñanza de la Anatomía Normal se ha visto enriquecida por la incorporación de otras metodologías, o la aplicación de conceptos provenientes de la Clínica y la Imágenología, con lo que se logra una mayor comprensión e integración de los conocimientos ${ }^{(1)}$. La incorporación de nuevas tecnologías aplicadas a imágenes han permitido la visualización del cuerpo humano en tres dimensiones y su transformación en un modelo físico conocido como "prototipo rápido" ${ }^{2,3)}$. Si a estos prototipos también conocidos como "biomodelos" le sumamos la posibilidad de confeccionarlos en materiales sintéticos de diferente color y consistencia, nos permitirá objetivar con facilidad las malformaciones o tumores y tendremos la posibilidad de contar con un instrumento de docencia $y$ entrenamiento clínico-quirúrgico de enorme valor ${ }^{(4)}$.

\section{Objetivo}

El objetivo de este trabajo es desarrollar un "prototipo rápido" en material sintético que replique con exactitud los detalles anatómicos con la finalidad de ejercitar la docencia y el entrenamiento quirúrgico en Pediatría.

\section{Material y Métodos}

Caso clínico: Paciente de un año de edad con síndrome de dificultad respiratoria. Durante el examen endoscópico con broncoscopio rígido se halló una compresión de la pared anterolateral derecha de la tráquea distal y carina. Los estudios angiotomográficos confirmaron la presencia de una malformación vascular con arco aórtico a la derecha y arteria subclavia derecha aberrante que surgía de un divertículo de Kommerell. Con la finalidad de analizar la conducta más adecuada, se solicitó la confección de un prototipo rápido a escala 1:1 que simulara una condición idéntica a la topografía torácica del paciente.

Técnica de imágenes: El paciente fue evaluado con tomógrafo multicorte de 40 cadenas de detectores con modos de adquisición isotrópicos. Se utilizaron protocolos de baja radiación con espesor de corte de $0,6 \mathrm{~mm}$ con matriz de $512 \mathrm{x}$ 512 sin angulación del "gantry". Se obtuvieron imágenes 2D con las que se efectuó la reconstrucción virtual en 3D para ser almacenadas en formato DICOM (digital imaging and communications in medicine).

Técnica de generación de prototipo: Se obtuvo una malla digital tridimensional que se optimizó respetando el modelo original a partir de la renderización de volúmenes. Esto permitió visualizar un conjunto de datos 3D originadas en cortes 2D captadas por Tomografía Axial Computada por medio de ingeniería inversa y técnicas de voxelización. Se exportó a formato compatible y se generó el código " $g$ " que se utilizó para controlar el hardware de producción. Se efectuó simulación digital y producción con técnica de modelo por deposición y fusión (MDF) utilizando ABS (Acrilonitrilo Butadieno Estireno). Este proceso se conoce actualmente como impresión 3D o prototipado rápido. La impresión del modelo se hizo efectiva reflejando las condiciones y características de cada una de las partes del organismo generando las cavidades internas y morfologías según detalle anatómico brindado por las imágenes. Finalmente se procedió a la validación del modelo prototipado comparándolo con las mediciones testigos tomadas del modelo virtual en $3 \mathrm{D}$.

\section{Resultados}

A partir de las imágenes virtuales (Figura № 1) se logró llevar a un "estado físico" lo que se conoce como prototipo rápido (Figuras № 2).

El modelo confeccionado en material plástico, replicó exactamente los defectos hallados en la tomografía y endoscopía, confirmando la presencia de la malformación vascular y su repercusión sobre el aparato respiratorio. Las mediciones del prototipo comparadas con el modelo virtual 3D proporcionó una construcción a escala 1:1 de diferentes órganos y sistemas de la estructura torácica generados en forma independiente. El sistema óseo conformado por las vértebras y sus respectivas costillas, permitió el ensamble del sistema cardiovascular y la vía aérea dentro de la cavidad torácica. En base a la información obtenida, se analizaron diferentes tácticas para la resolución del problema.

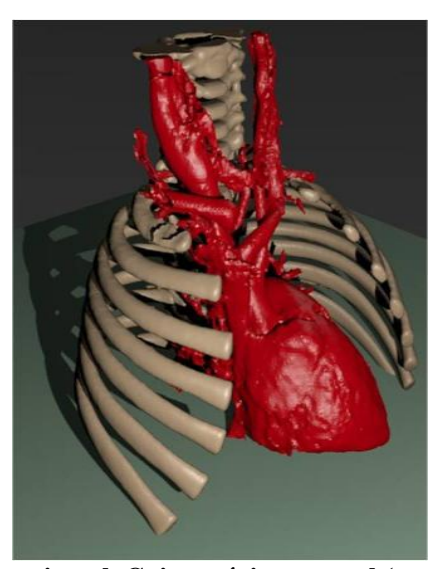

Figura 1: Prototipo virtual. Caja torácica normal (parcial), corazón y grandes vasos en paciente de 1 año de edad 


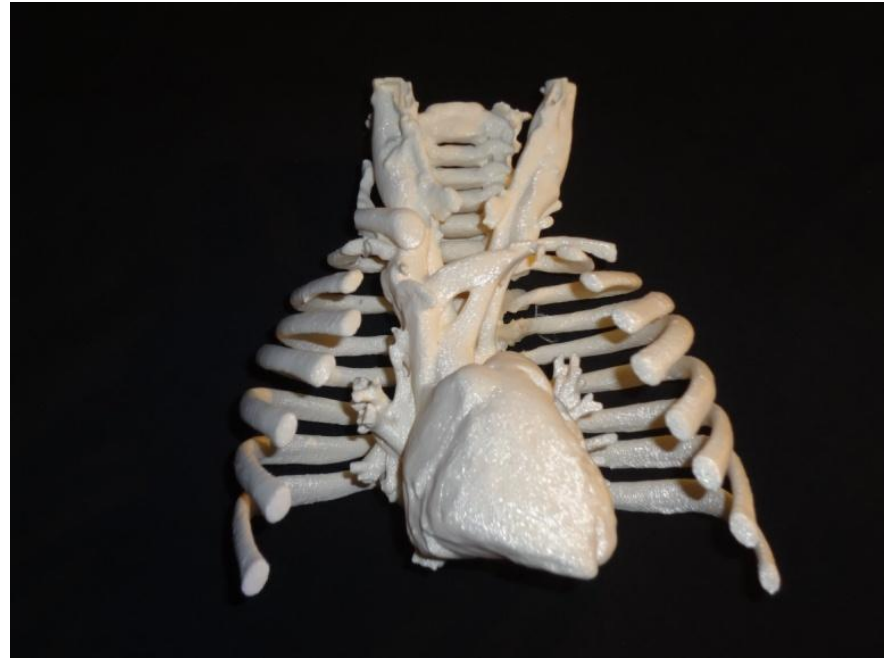

Figura 2: Estado físico del prototipo virtual construido en ABS. Caja torácica, corazón y grandes vasos en paciente de 1 año de edad

\section{Discusión}

La enseñanza de la Anatomía está incorporando información aportada por la Clínica y los estudios de diagnóstico más modernos especialmente la Imagenología. Se ha demostrado que cursos de anatomía de menor duración pero acompañados de información clínica, aportan un muy buen nivel de aprendizaje ${ }^{(1)}$. Esto induce a pensar que las imágenes virtuales $3 \mathrm{D}$, son una herramienta útil para la comprensión y fijación de los conocimientos anatómicos. Si a esto le sumamos la posibilidad de llevar a estado físico las imágenes 3D, podemos contar con un material didáctico aún no convencional en la docencia, pero que promete ser de gran utilidad ${ }^{(2,3,4)}$. El desarrollo de un prototipo rápido que muestra la forma y el contenido del cuerpo humano pediátrico es una herramienta de gran valor para el conocimiento anatómico normal en los primeros años de la vida. Si a esto le agregamos la posibilidad de una visión topográfica o de evaluar situaciones "patológicas" como la presencia de malformaciones o tumores, este instrumento podría adquirir gran valor para la docencia de grado y postgrado, brindando además la posibilidad de ejercitar la comparación con lo "normal". Cuando se realizan encuestas a alumnos y profesores referidas al entrenamiento sobre réplicas en modelos físicos en pacientes con tumores, se ha encontrado preferencia notable sobre los modelos virtuales ${ }^{(4)}$. Otra gran ventaja que aporta el prototipo es que las estructuras internas de los órganos son respetadas con máxima precisión. Esta "copia anatómica" permite comprender la relación con otros órganos y a la vez evaluar las condiciones intrínsecas y consecuencias de una anomalía anatómica como en el caso presentado. La posibilidad de trabajar sobre un prototipo, permite proponer un plan de tratamiento más ajustado a la realidad. Si bien las imágenes virtuales en 3D ofrecen la posibilidad de analizar partes anatómicas desde varias posiciones, la disponibilidad de un prototipo rápido despertaría el interés del recurso humano en formación y a la vez facilitaría el entrenamiento del equipo quirúrgico.

La técnica de construcción de este prototipo rápido se basó en depósito y fusión de materiales, en este caso ABS que es una variedad de plástico que le da una consistencia sólida y se encuentra dentro de las más utilizadas ${ }^{(3)}$. La construcción de los prototipos rápidos podría ser realizada con diversos tipos de materiales de diferente flexibilidad, consistencia y costos variables.

Hay numerosas áreas de la medicina que podrían beneficiarse con este modelo de tecnología innovadora. Una de las áreas en las que más se ha desarrollado esta tecnología, es la reconstrucción cráneo-facial, la reconstrucción mandibular cuando la anatomía está muy distorsionada o cuando hay ausencia de tabla ósea aplicando prótesis de titanio hechas con exactitud a partir de imágenes $3 D{ }^{(5,6,7,8)}$. En el área de la ortopedia los resultados también parecen alentadores ensayando procedimientos de artroplastia total de cadera con máxima seguridad ${ }^{(9)}$. Finalmente y para agregar un punto más de interés en el amplio espectro de aplicaciones se destaca su uso en el análisis de situaciones controvertidas en el ámbito de la medicina forense ${ }^{(10)}$.

\section{Conclusión}

El prototipo rápido es el estado físico de una imagen virtual 3D confeccionado en material sintético que permitiría el ejercicio de la docencia y el entrenamiento del equipo quirúrgico en Pediatría.

Agradecimientos A los profesionales de la Facultad de Arquitectura Urbanismo y Diseño, Facultad de Ciencias Médicas de la Universidad Nacional de Córdoba y a la Fundación FIDEIAR por el empeño en el trabajo conjunto para lograr el desarrollo de un prototipo rápido y llevar al estado físico el proyecto con imágenes virtuales en 3D. 


\section{Bibliografía}

1. Rizzolo LJ, Rando WC, O’Brien MK, et al. Design, implementation, and evaluation of an innovative anatomy course. Anat Sci Educ 2010;3:109-20.

2. Li J, Nie L, Li $Z$ et al. Maximizing modern distribution of complex anatomical spatial information: $3 D$ reconstruction and rapid prototype production of anatomical corrosion cast of human specimens. Anat Sci Educ. 2012;5:330-39.

3. Murugesan K, PA, Sharma SK, et al. Comparative Evaluation of Dimension and Surface Detail Accuracy of Model Produced by Three Different Rapid Prototype Techniques. J Indian Prosthodont Soc 2012;12:16-20.

4. Challoner A, Erolin C. Creating pathology models from MRI data: a comparison of virtual $3 D$ modeling and rapid prototyping techniques. $J$ Vis Commun Med 2013;36:11-19.

5. Morrison DA, Guy DT, Day RE, et al. Simultaneous repair of two large cranial defects using rapid prototyping and custom computerdesigned titanium plates: a case report. Proc Inst Mech Eng H. 2011;225:1108-12

6. Bullock $P$, Dunaway D, McGurk $L$, et al. Integration of image guidance and rapid prototyping technology in craniofacial surgery. Int J Oral Maxillofac Surg 2013;42:970-3.

7. Hanasono MM, Skoracki RJ. Computer-assisted design and rapid prototype modeling in microvascular mandible reconstruction. Laryngoscope 2013;123:597-604.

8. Taft RM, Kondor S, Grant GT. Accuracy of rapid prototype models for head and neck reconstruction. J Prosthet Dent 2011;106:399408.

9. Faur C, Crainic N, Sticlaru C. Rapid prototyping technique in the preoperative planning for total hip arthroplasty with custom femoral components. Wien Klin Wochenschr. 2013;125:144-9

10. Wozniac K, Rzepecka-Wozniac E, Moskala A, et al. Weapon identification using antemortem computed tomography with virtual 3D and rapid prototyped modeling: a report in a case of blunt force head injury. Forensic Sci Int 2012;222: e29-32. 